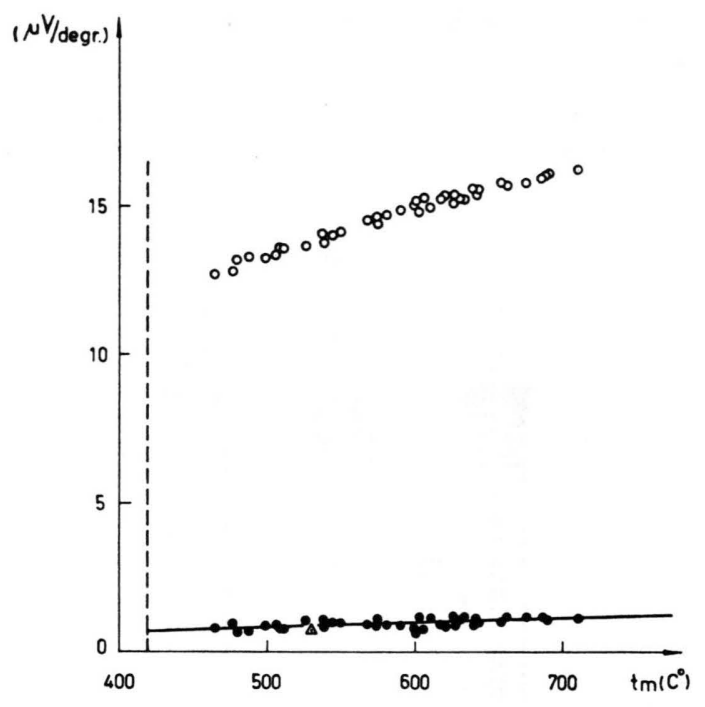

Fig. 2. Temperature dependence of thermoelectric powers. 0 : measured thermoelectric power $\varepsilon \mathrm{Zn}, \mathrm{w}, \bullet$ : calculated absolute thermoelectric power $S_{\mathrm{Zn}}, \triangle: S_{\mathrm{Zn}}$ reported by BRADLEY ${ }^{3}$. and it is thus possible to calculate $S_{\mathrm{Zn}}=S_{\mathrm{W}}-\varepsilon_{\mathrm{Zn}, \mathrm{W}}$, see Fig. 2. A least squares fit gives

$S_{\mathrm{Zn}}=(0.19 \pm 0.17)+(1.30 \pm 0.28) \cdot 10^{-3} t_{\mathrm{m}} \mu \mathrm{V} / \mathrm{degr}$.

where $t_{\mathrm{m}}$ is the mean temperature in ${ }^{\circ} \mathrm{C}$. The quoted errors are the standard deviations of the coefficients, while the standard deviation of $S_{\mathrm{Zn}}$ is $0.12 \mu \mathrm{V} / \mathrm{degr}$. Our results are in good agreement with the single measurement at $530{ }^{\circ} \mathrm{C}$ reported by BRADLEX ${ }^{3}$, but differ considerably from elder measurements ${ }^{\mathbf{1 1}}$.

PÉlABon ${ }^{12}$ reports an investigation of the thermocell $\mathrm{Pt} / \mathrm{Zn}$ (liq.) $/ \mathrm{Pt}$, but we found that platinum dissolves too rapidly in liquid zinc to allow for reliable measurements.

We are indebted to Dr. A. Kvist for stimulating discussions. Financial support was provided by the Swedish Technical Research Council.

11 E. G. Linder, Phys. Rev. 29, 554 [1927].

12 H. PÉlabon, Ann. Phys., Paris 13, 169 [1920].

\section{Die Diffusion von Tritium in einer Silber-Lithium-Legierung *}

H. Migge, K. Wagener und K. E. Zimen

Hahn-Meitner-Institut für Kernforschung Berlin

(Z. Naturforschg. 20 a, 1082-1084 [1965] ; eingegangen am 2. Juli 1965)

\section{Fragestellung}

In Lithium-haltigen Festkörpern lassen sich durch Neutronenbestrahlung leicht relativ hohe Konzentrationen an Tritium und Helium erzeugen durch die Kernreaktion ${ }^{6} \mathrm{Li}(\mathrm{n}, \alpha){ }^{3} \mathrm{H}$. Wir nutzten diese Tatsache aus, um die Diffusion dieser Gase in Silber zu untersuchen, dem natürliches Lithium zulegiert war. Das Interesse an derartigen Versuchen ergab sich, nachdem in der Kinetik des Austritts von Spaltedelgasen aus neutronenaktivierten Festkörpern in zahlreichen Fällen Abweichungen vom theoretisch zu erwartenden Verlauf beobachtet worden waren ${ }^{1}$.

Zum Nachweis des aus den Proben ausgetretenen Gases war eine kontinuierlich arbeitende massenspektrometrische Methode vorgesehen worden, da wir zunächst glaubten, den Austritt beider Gase gleichzeitig verfolgen zu können. Es zeigte sich je-

* Nr. 23 der Reihe: Edelgasdiffusion in Festkörpern. doch bald, daß eine mit unserer Apparatur meßbare Ausscheidungsrate an Helium offenbar erst aus der Schmelze stattfindet, während der Wasserstoffaustritt schon bei tiefen Temperaturen gut meßbar ist. In der vorliegenden Arbeit wird daher zunächst über die Tritium-Diffusion berichtet.

\section{Experimentelles}

Die Proben bestanden aus Silber mit 20 At.-Proz. natürlichem Lithium. Abmessungen $12 \times 7 \times 2 \mathrm{~mm}^{3}$. Es wurden weichgeglühte Proben verwandt sowie solche, die um ca. $50 \%$ kaltverformt waren. Die Rekristallisation der kaltverformten Proben tritt bei einer Aufheizgeschwindigkeit von $2^{\circ} / \mathrm{min}$ zwischen 200 und $250^{\circ} \mathrm{C}$ ein, wie sich aus dem Härte-Temperatur-Verlauf ergibt.

Die Bestrahlung der Proben erfolgte im Berliner Reaktor BER bei einem thermischen Neutronenfluß von $3,1 \cdot 10^{11} / \mathrm{cm}^{2} \mathrm{~s}$ bis zu einer Dosis von $3,1 \cdot 10^{16}$ pro $\mathrm{cm}^{2}$. Als Folge der genannten Kernreaktion besaßen die Proben danach eine errechnete Spaltgaskonzentration (unter Berücksichtigung der Flußdepression sowie der Neutronenselbstabsorption) von $1,2 \cdot 10^{16}$ Atome $/ \mathrm{cm}^{3}$ an ${ }^{4} \mathrm{He}$ bzw. ${ }^{3} \mathrm{H}$ (entsprechend einer Gitterkonzentration von $0,21 \cdot 10^{-6}$ ).

1 Vgl. z. B.: T. Lagerwall u. P. Schmeling, Ber. Hahn-MeitnerInstitut HMI-B 27 [1963]. 
Das Ausheizen der Proben erfolgte im Hochvakuum $\left(<10^{-6}\right.$ Torr $)$, wobei sie sich in einem zylindrischen Eisentiegel befanden, der induktiv von außen geheizt wurde. Es wurde stets im offenen System gearbeitet (d. h. bei laufender Pumpe) und die jeweils aus der Probe austretende Gasrate beobachtet. Meßbereich: bis ca. $700{ }^{\circ} \mathrm{C}$; die Proben konnten während der Messung auch geschmolzen werden.

Wir führten Entgasungsversuche bei konstanter Temperatur aus (Aufheizzeit der Probe nach Einwerfen in den vorgeheizten Tiegel: ca. $2 \mathrm{~min}$ ) sowie bei stetig steigender Temperatur (Aufheizgeschwindigkeiten von 1 bis $4^{\circ} / \mathrm{min}$ ). Die Austrittsrate des Tritiums wurde von etwa $200{ }^{\circ} \mathrm{C}$ an meßbar. Bei den Versuchen unter kontinuierlich gesteigerter Temperatur blieb sie bis etwa $550{ }^{\circ} \mathrm{C}$ beobachtbar; danach waren die Proben jeweils leergelaufen. Die Glühzeiten betrugen 1 bis 5 Stunden.

Die Messung der Entgasungsrate erfolgte kontinuierlich auf massenspektrometrischem Wege mit Hilfe eines Omegatrons (Fa. L e y b o ld, Köln). Die Auflösung des Gerätes reicht aus, um die Ionen $\mathrm{HT}^{+}$ und ${ }^{4} \mathrm{He}^{+}$unterscheiden zu können. Das aus dem Inneren an die Probenoberfläche gelangende Tritium bildet dort (infolge des etwa $10^{3}$-mal größeren Wasserstoffpartialdrucks im Vakuumsystem) HT, dessen Partialdruck im System beobachtet wird und ein proportionales $\mathrm{Ma} \beta$ für die Austrittsrate an Tritium aus der Probe ist. Eine ausführliche Beschreibung des Aufbaues und der Eichung dieser Apparatur erfolgt später.

\section{Auswertung der Messungen und Ergebnisse}

Bei allen Versuchen konnte die Abgabe des gesamten Tritiums aus der Probe beobachtet werden, falls sie bei hinreichend hoher Temperatur bzw. hin. reichend lange geglüht wurde. Eine Retention, wie sie für Edelgase in Kernbrennstoffen beobachtet wird (vgl. 1), findet also beim Wasserstoff in diesem System nicht statt.

Aus den Messungen bei konstant gehaltener Temperatur wurde der Diffusionskoeffizient des Tritiums in der Legierung aus dem zeitlichen Abfall der Austrittsrate bestimmt. Der beobachtete Verlauf entspricht der zu erwartenden Kinetik für das „Auslaufen“ einer Probe ${ }^{2}$, so daß im gesamten Konzen-

2 Vgl. W. Inthoff u. K. E. Zimen, Trans. Chalmers Univ. Technol. (Göteborg) No. 176, 1 [1956]. - T. Lagerwall u. K. trationsbereich eine konstante Gasbeweglichkeit in der Probe vorzuliegen scheint. Die einzige signifikante Diskrepanz zwischen Beobachtung und Erwartung auf Grund des einfachen Diffusionsmodells besteht darin, daß die Ausscheidung zunächst nur zögernd beginnt: während der ersten 15-25 Minuten wächst die insgesamt ausgetretene Gasmenge nicht proportional $t^{1 / 2}$, sondern etwa proportional $t^{3 / 2}$, bis die Gasrate danach den „normalen“ Wert erreicht hat. Dieses Verhalten konnte bis zu Temperaturen von ca. $390{ }^{\circ} \mathrm{C}$ beobachtet werden.

Die Versuche bei monoton steigender Temperatur ergaben naturgemäß eine zunächst steigende und dann wieder abfallende Gasrate. Bei der Auswertung dieser Versuche wurde angenommen, daß reine Gitterdiffusion vorliegt und kein Einfang von Gas innerhalb der Probe (traps) stattfindet. Aus jedem Ausheizprozeß erhält man dann ein Arrhenius-Diagramm des (ggf. effektiven) Diffusionskoeffizienten des Tritiums über den beobachteten Temperaturbereich (vgl. hierzu die Diskussion) .

Die Ergebnisse beider Ausheizmethoden (konstante bzw. monoton steigende Temperatur) sind in bezug auf die daraus ermittelten Diffusionskoeffizienten im Rahmen der Meßgenauigkeiten gleich. Es sind zwei Temperaturbereiche zu unterscheiden, in denen ein auffallend unterschiedliches Verhalten bezüglich der Tritiumabgabe zu beobachten ist:

(a) für Temperaturen über ca. $370{ }^{\circ} \mathrm{C}$ :

$$
\begin{aligned}
D_{0} & =1,1 \cdot 10^{-2} \mathrm{~cm}^{2} / \mathrm{s}, \\
Q & =0,49 \mathrm{eV} \text { oder } 11,5 \mathrm{kcal} / \mathrm{mol}, \\
\text { somit } \quad D & =1,1 \cdot 10^{-2} \exp [-0,49 / k T] \mathrm{cm}^{2} / \mathrm{s} .
\end{aligned}
$$

(b) für Temperaturen unter ca. $350{ }^{\circ} \mathrm{C}$ wird eine wesentlich stärkere Temperaturabhängigkeit beobachtet, die einer Aktivierungsenergie von $Q=1,5 \mathrm{bis} 1,9 \mathrm{eV}$ oder $35 \mathrm{bis} 45 \mathrm{kcal} / \mathrm{mol}$ entspricht.

Die Rekristallisation kaltverformter Proben, die (wie oben erwähnt) bei kontinuierlichem Aufheizen zwischen 200 und $250{ }^{\circ} \mathrm{C}$ abläuft, hat keinen erkennbaren Einfluß auf die Entgasungskinetik. Beim Schmelzen der Proben tritt eine diskontinuierliche, stoßartige Gasgabgabe auf. Wir vermuten, da $\beta$ es sich dabei um aus der Schmelze aufsteigende Heliumblasen handelt.

E. Zimen, Ber. Hahn-Meitner-Institut HMI-B 25 [1963]. 


\section{Diskussion}

Unsere Ergebnisse für Temperaturen über ca. $370^{\circ} \mathrm{C}$ zeigen ein sehr ähnliches Verhalten des Tritiums, wie es für die Diffusion von ${ }^{1} \mathrm{H}$ in reinem Silber im Temperaturbereich zwischen 388 und $600{ }^{\circ} \mathrm{C}$ von Eichenauer, Künzig und Pebler ${ }^{3}$ gefunden wurde:

$$
D=2,82 \cdot 10^{-3} \exp [-0,32 / k T] \mathrm{cm}^{2} / \mathrm{s} .
$$

Die merkwürdige Erscheinung, daß die Ausscheidungsrate an Gas bei tiefen Temperaturen ( $<$ ca. $350{ }^{\circ} \mathrm{C}$ ) eine wesentlich stärkere Temperaturabhängigkeit zeigt als bei höheren $\left(>\right.$ ca. $370{ }^{\circ} \mathrm{C}$ ), legt die Vermutung nahe, daß es zweier Reaktionsschritte bedarf, bevor das Gas den Kristall verlassen kann. Am wahrscheinlichsten erscheint folgender Mechanismus :

Infolge Ubersättigung an Wasserstoff findet eine innere Ausscheidung statt, vermutlich von molekularem Wasserstoff. Danach gibt es drei energetisch ausgezeichnete Zustände des Wasserstoffs innerhalb der Probe: (1) innere Ausscheidung (= energetisch tiefster Zustand), (2) auf Zwischengitterplätzen, (3) beweglich (= zum Platzwechsel innerhalb des Gitters fähig). Die im oberen Temperaturbereich beobachtete Aktivierungsenergie wäre danach als Platzwechselenergie [Übergang (2) nach (3)] zu deuten, während die große Aktivierungsenergie im unteren Temperaturbereich im wesentlichen der Lösung des ausgeschiedenen Wasserstoffs im Kristall zuzuschreiben wäre.

Wie von Gaus vor kurzem gezeigt werden konnte ${ }^{4}$, ergibt sich bei Annahme zweier stabiler, energetisch nicht gleichwertiger Positionen [vgl. die Zustände (1) und (2) ] ferner, daß die vom Kristall nach außen abgegebene Gasmenge dann zu Beginn des Versuchs mit $t^{3 / 2}$ wächst, sofern der Hauptteil des Gases zu Beginn des Versuches im Zustand (1) vorliegt. Da die Gasabgabe zunächst tatsächlich propor-

3 W. Eichenauer, H. Künzig u. A. Pebler, Z. Metallk. 49, 220 [1958].

${ }^{4}$ H. Gaus, Z. Naturforschg. 20 a [1965], im Druck.

5 C. E. Ells u. W. Evans, Trans. Met. Soc. AIME 227, 438 [1963].

6 W. Carnuth, Z. Angew. Phys. 15, 291 [1963]. tional $t^{3 / 2}$ verläuft (s. o.), erscheint dies als Stütze für den angenommenen Ausscheidungsmechanismus.

Die Existenz von molekularem Wasserstoff in der Probe, der allein eine entsprechend große Lösungsenthalpie besitzen würde, müßte im vorliegenden Fall erst noch nachgewiesen werden. In Aluminium wurden Blasen bereits bei einer Gitterkonzentration des Wasserstoffs von $0,16 \cdot 10^{-6}$ lichtmikroskopisch nachgewiesen ${ }^{5}$, die beim Tempern der Proben wieder verschwanden. Diese Beobachtung würde eine innere Ausscheidung von molekularem Wasserstoff erweisen.

Im Gegensatz dazu steht eine Interpretation von Carnuth ${ }^{6}$, der in kaltverformtem Kupfer, das in einer Wasserstoffatmosphäre mit Tritium beladen wurde, eine gegenüber unverformten Proben stark verlangsamte (effektive) Wasserstoffdiffusion beobachtete. Er führt diese Hemmung darauf zurück, daß die bei der Verformung entstehenden Gitterfehler als Haftstellen für Wasserstoffatome wirken und gibt die Bindungsenergie der Atome an die Haftstellen zu 1,9 eV an. Diese Deutung erscheint unwahrscheinlich, da die Bindungsenergie von Zwischengitterfremdatomen an Gitterdefekten im allgemeinen kleiner als $1 \mathrm{eV}$ gefunden wird ${ }^{7}$.

Für die Argonabgabe aus KCl-Einkristallen fanden Richter und ZimeN ${ }^{8}$ ein ganz analoges Verhalten (starke Temperaturabhängigkeit im unteren, schwache im oberen Temperaturbereich sowie verzögerter Beginn der „normalen“ Abgabekinetik). Sie vermuteten bereits, daß dieses Phänomen mit der Strahlenschädigung des Kristalls nicht in Zusammenhang steht, sondern andere Ursachen haben müsse. Ein dem hier diskutierten ähnlicher Mechanismus könnte auch dort vermutet werden.

Diese Arbeit wurde teilweise im Rahmen des Joint Euratom/United States Program (Kontrakt Nr. 028-64-1-TEE R+D) durchgeführt. Herrn Dr. H. Gaus danken wir für zahlreiche Diskussionen.

7 Vgl. z. B.: A. C. Damask u. R. A. Arndt, BNL-7696 [1963]. - R. E. Smallman, Modern Physical Metallurgy, Butterworths, London 1963, S. 250.

8 A. K. H. Richter u. K. E. Zimen, Z. Naturforschg. 20 a, 666 [1965]. 